\title{
Grasping-Related Functional Magnetic Resonance Imaging Brain Responses in the Macaque Monkey
}

\author{
Koen Nelissen ${ }^{1,2}$ and Wim Vanduffel ${ }^{1,2}$ \\ ${ }^{1}$ Laboratorium voor Neuro-en Psychofysiologie, Katholieke Universiteit Leuven Medical School, 3000 Leuven, Belgium, and ${ }^{2}$ Athinoula A. Martino's Center \\ for Biomedical Imaging, Massachusetts General Hospital, Harvard Medical School, Charlestown, Massachusetts 02129
}

Research in recent decades has suggested the existence of a dedicated brain network devoted to the organization and execution of grasping, one of the most important and skilled movements of primates. Grasping an object requires the transformation of intrinsic object properties such as size, orientation, and shape into an appropriate motor scheme shaping the hand. Although electrophysiological recordings in the monkey model have proven invaluable for gaining insights into the neuronal substrate underlying this complex behavior, knowledge concerning the existence and organization of a similar system in the human brain is derived mainly from imaging studies. Here, we present for the first time functional magnetic resonance imaging (fMRI) of brain activity while macaque monkeys performed reaching and grasping movements in a 3 tesla MR scanner. Grasping in the dark (compared with reaching) yielded significant activations in anterior intraparietal area and ventral premotor area F5, in addition to area PFG in the rostral inferior parietal lobule, somatosensory areas (SI, SII, area 5), and the hand field of F1. Whole-brain macaque fMRI motor studies will be instrumental in establishing possible homologies concerning grasping organization in the human and monkey brains, bridging the gap between human imaging and monkey electrophysiology.

\section{Introduction}

Grasping involves transporting the arm and shaping the hand to match the shape and size of the object. Based on lesion data from both patients and monkeys showing that grip formation can be affected while leaving reaching intact (Gallese et al., 1994; Jeannerod et al., 1994; Binkofski et al., 1998; Fogassi et al., 2001), it was originally proposed that grasping behavior (for review, see Culham and Valyear, 2006; Brochier and Umiltà, 2007; Castiello and Begliomini, 2008; Filimon, 2010; Grafton, 2010) can be dissociated into separate reach and grip components. According to this view, grasping is organized by a lateral parietofrontal circuit involving anterior intraparietal area (AIP) and premotor F5 (Rizzolatti et al., 1988; Jeannerod et al., 1995) and reaching by a more medial parietofrontal circuit including medial intraparietal area (MIP) [also referred to as the parietal reach region (PRR)], area V6A, and dorsal premotor area F2 (Kalaska et al., 1997; Fattori et al., 2001; Buneo et al., 2002; Gregoriou and Savaki, 2003).

\footnotetext{
Received Feb. 4, 2011; revised March 23, 2011; accepted April 15, 2011.

Author contributions: K.N. and W.V. designed research; K.N. performed research; K.N. analyzed data; K.N. and W.V. wrote the paper.

This work was supported by the Queen Elisabeth Foundation, Program Financing/10/008, Interuniversity Attraction Pole 6/29, Geconcerteerde Onderzoeksactie/10/19, Hercules II funds, Fonds Wetenschappelijk Onderzoek (FW0) Vlaanderen G.0.622.08 and G.0.593.09, National Institute of Neurological Disorders and Stroke Grant 1R21 NS064432, and National Science Foundation Grant BCS-0745436. K.N. is a postdoctoral research fellow of the FWO. We thank A. Coeman, W. Depuydt, M. De Paep, M. Docx, C. Fransen, P. Kayenbergh, Dr. H. Kolster, G. Meulemans, Dr. R. Peeters, I. Puttemans, C. Van Eupen, and S. Verstraeten for technical assistance, Dr. D. Stoianovici from Johns Hopkins Medicine for providing the Pneustep MR-compatible motor, and Drs. G. Luppino and S. Raiguel for valuable comments on this manuscript.

Correspondence should be addressed to Koen Nelissen, Laboratorium voor Neuro-en Psychofysiologie, Katholieke Universiteit Leuven Medical School, Herestraat 49, bus 1021, 0\&N2 Campus Gasthuisberg, 3000 Leuven, Belgium. E-mail: koen.nelissen@med.kuleuven.be.

DOI:10.1523/JNEUROSCI.0623-11.2011

Copyright $\odot 2011$ the authors $\quad 0270-6474 / 11 / 318220-10 \$ 15.00 / 0$
}

Human imaging studies also support this view, showing grasp-related responses in the anterior portion of human intraparietal sulcus (Grafton et al., 1996; Faillenot et al., 1997; Binkofski et al., 1998; Culham et al., 2003; Frey et al., 2005), whereas reaching activates medial intraparietal and superior parietooccipital cortex (Connolly et al., 2003; Prado et al., 2005; CavinaPratesi et al., 2010).

This dichotomic view, however, has recently been challenged, since both lesion (Battaglini et al., 2002) and single-cell data (Raos et al., 2004; Fattori et al., 2009, 2010) in the macaque argue against a strict separation of dedicated reach and grasp circuits. These studies provide evidence for involvement of areas V6A and F2 in organizing specific aspects of grasping behavior (i.e., wrist orientation and grip posture).

Using functional MRI in the macaque, we investigated the brain regions involved in organizing grasping behavior by contrasting grasping with reach-only movements. For the first time, we present whole-brain fMRI data derived from macaques performing grasping and reaching tasks while maintaining central fixation, using a magnet-compatible computer-controlled rotor system that presented differently sized and shaped objects to the monkey. Both motor tasks were performed in the dark to ensure that $\mathrm{fMRI}$ activations were attributable to the motor aspects of the task and were not attributable to object observation.

Grasping, compared with reaching, yielded significant fMRI activations in ventral premotor F5, parietal area AIP, the hand region of F1, and various portions of the somatosensory cortex (SI, SII, areas 5 and 7). In addition to confirming the roles of AIP and F5 in grasping control, these data also point to the strong engagement of primary and secondary somatosensory cortex when grasping relies on memory-based information and haptic feedback. 

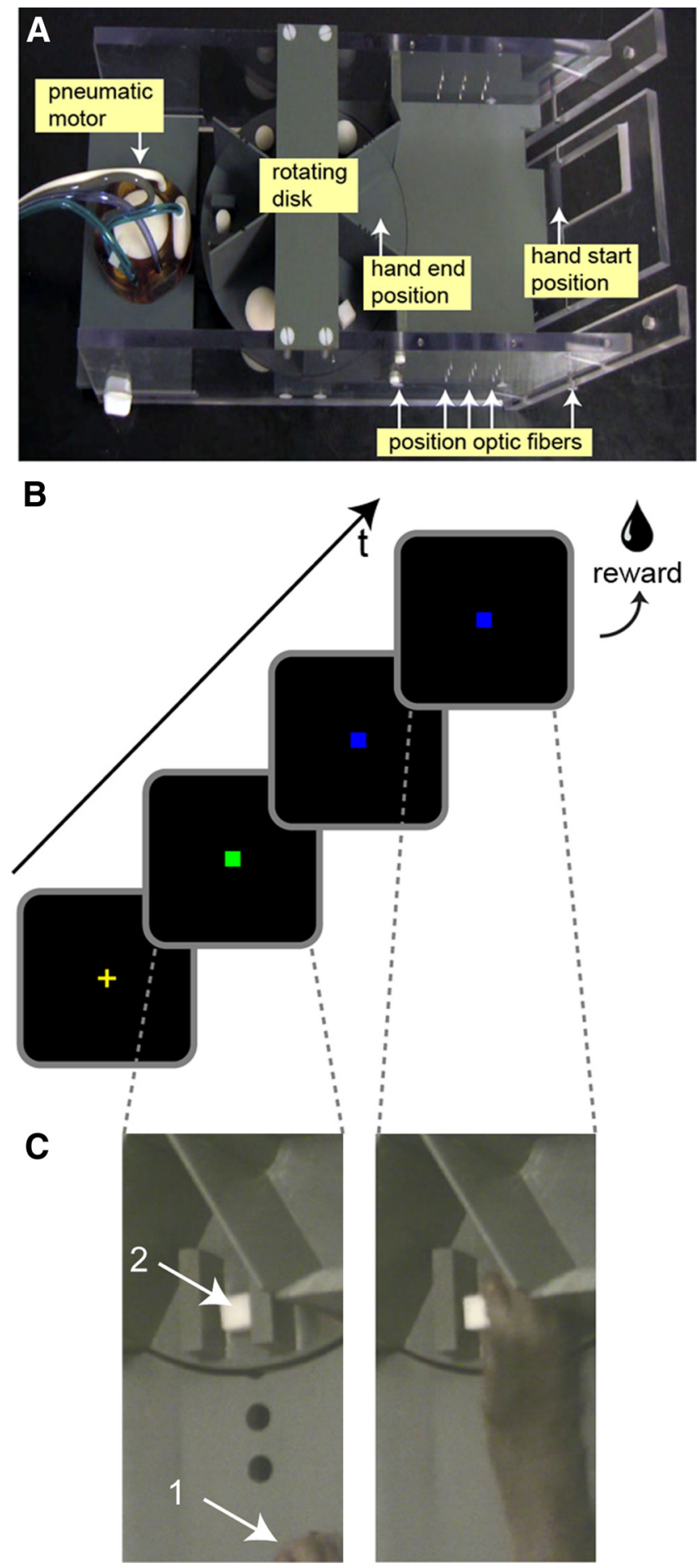

Figure 1. Grasping setup and experimental design. $\boldsymbol{A}$, The MR-compatible device for studying grasping consists of a horizontal rotating disk containing five different objects. The objects are connected through a plastic shaft to a small plastic weight under the disk, allowing the objects to fall back in place after being grasped and lifted by the monkey. One slot on the disk is left empty for the reach-only trials. The rotating disk is powered by an MR-compatible pneumatic stepper motor and connected through a belt and gear system below the disk. Start and end positions for the hand trajectory during the grasping and reaching trials are indicated. Hand and object position, as well as speed of the transport phase of the hand during the grasping and reaching trials, is monitored through the use of several optic switches along the path. $B, C$, Visual cues used during the grasping and reaching trials. Grasping and reach-only tasks were performed in the dark. To initiate the trial, the monkey had to place its right hand in the start position ( $\boldsymbol{C}$, arrow 1 ) and fixate a small green fixation point displayed centrally on a screen $(\boldsymbol{B})$. After fixating for a random period of time ( $500-1500 \mathrm{~ms}$ ) while keeping its hand in the start position, the fixation point changes to blue. This color signals to the monkey that he could reach
Bridging the gap between human fMRI and macaque singleunit recordings, monkey fMRI motor studies will be useful for gaining better insights into the complex organization and control of prehension behavior in the brain and will help to establish possible homologies between primate species.

\section{Materials and Methods}

\section{Subjects}

Two male (M1, M2) rhesus monkeys (Macaca mulatta; $3-5 \mathrm{~kg}$; $3-5$ years of age) participated in the experiments. All animal care and experimental procedures met the national and European guidelines and were approved by the ethical committee of the Katholieke Universiteit Leuven Medical School. The details of the surgical procedures, training of monkeys, image acquisition, eye monitoring, and statistical analysis of monkeys scans have been described previously (Vanduffel et al., 2001; Nelissen et al., 2005, 2006; Ekstrom et al., 2008) and will be reviewed here only briefly.

\section{Fixation training}

The monkey subjects sat in a sphinx position in a plastic monkey chair directly facing a LCD screen. During initial training, they were required to maintain fixation within a $2 \times 2^{\circ}$ window centered on a red dot $\left(0.35 \times 0.35^{\circ}\right)$ in the middle of the screen. Eye position was monitored at $120 \mathrm{~Hz}$ through the pupil position and corneal reflection (Iscan). During this training phase, the monkeys were rewarded (fruit juice) for fixating the small red dot within the fixation window for long periods (up to several minutes).

\section{Rotating grasping device}

For the grasping experiments, we custom built a magnetic resonance (MR)-compatible turntable or rotating disk simultaneously holding five different objects (Fig. 1A), which was attached to the front of the monkey fMRI chair (for an MR-compatible grasping apparatus used in human fMRI motor studies, see Culham et al., 2003). The turntable was powered through a gear and belt system, driven by an MR-compatible pneumatic stepper motor (Stoianovici et al., 2007) built by the Urology Robotics Department of Johns Hopkins Bayview Medical Center (Baltimore, MD) (http://urobotics.urology.jhu.edu/projects/PneuStep). This system was computer controlled and could be triggered by the scanner. The disk could rotate with a speed of $15 \%$. The sixth slot on the rotating disk was empty and was used for the motor control task (reach-only). Each of the objects was connected through a shaft to a small plastic weight below the disk. This allowed the objects to fall back into place once the monkey subject released the object after completing the grasping movement. Optical fibers were used to monitor the position of the objects. On one of the five object slots on the disk were glued two elongated plastic bars. A small object (cube or cylinder) could be positioned between these bars (Fig. $1 C$ ) so that the object could be grasped and lifted only with a precision grip (thumb and index finger).

\section{Motor tasks}

The monkeys were trained to perform two different motor tasks, a reachand-grasp task and a reach-only task. Two movies (Nelissen_grasping task.mp4 and Nelissen_reaching_task.mp4), showing examples of the reach-and-grasp and reach-only tasks, can be viewed or downloaded from our laboratory website (http://neuroserv.med.kuleuven.be/ downloadarea/stimuli.php). Since we were interested in investigating the motor-related response during grasping, both motor tasks were per-

$\leftarrow$

and, depending on the current task, either grasp and lift (5 $\mathrm{mm}$ upward) the object on the rotating disk ( $\boldsymbol{C}$, arrow 2 ) or place its open hand on the empty slot of the disk (Fig. 2F). After holding the object for $530 \mathrm{~ms}$ during the grasping trials (or leaving its open hand on the disk for 530 ms during the reach-only trials), a juice reward was given. Failure to either place the hand in the correct start position at the beginning of the trial, failure to grasp and lift the object (or place the hand) within $2000 \mathrm{~ms}$ after onset of the blue fixation point, or breaking fixation in general during a trial would result in an abort during which a yellow cross was presented. A new trial (green fixation point) started only if the monkey fixated the fixation point and placed its hand in the correct starting position. 
formed in the dark. The objects were never illuminated and monkeys relied on haptic and memory feedback to perform the motor tasks. This ensured that visibility of the graspable objects or moving arm did not confound the brain activations, since many visuomotor neurons in AIP and F5 also discharge during the mere presentation of an object, without requiring a grasping movement (Murata et al., 2000; Rizzolatti and Luppino, 2001; Raos et al., 2006).

The reach-only task served as a motor control task, since it contains the same reaching phase as is present in the reach-and-grasp task, but without the hand shaping phase required for grasping. Both monkeys were trained for several months on the motor tasks before scanning. Video monitoring was used during the daily training sessions.

\section{Reach-and-grasp task}

The grasping task consisted of the following sequence of events: A trial started when the monkey placed its hand in the start position (Fig. $1 \mathrm{~A} ; \mathrm{C}$, position 1 indicated with white arrow) and fixated on a green fixation point displayed centrally on the screen in front of him (Fig. $1 B$ ). If the monkey removed its hand or stopped fixating before a certain random time (between 500 and $1500 \mathrm{~ms}$ ), the trial was aborted and a yellow cross was displayed until he again placed its hand at the starting position. After a random fixation time (500-1500 ms), the green fixation point would change to blue, indicating to the monkey that he could now reach and grasp the object with its right hand (Fig. $1 C$, position 2 indicated with white arrow). Failure to reach and grasp the object within $2000 \mathrm{~ms}$ would result in an aborted trial. After the monkey had grasped the object, he was required to lift it for $5 \mathrm{~mm}$ and hold it in that position for $530 \mathrm{~ms}$ (Fig. $1 B, C)$ to receive a juice reward. After delivery of the reward, a new trial started (green fixation point) as soon as the monkey returned his hand to the initial starting position while keeping fixation. At the start and end position of the reaching trajectory (Fig. $1 A$ ), as well as at three locations along the hand/arm trajectory (Fig. $1 \mathrm{~A}$, position optic fibers), we positioned optic fiber cables, which allowed us to track the location of the monkeys' hand/arm while performing the task. The optic fibers also allowed us to record and control the timing of the execution of the motor tasks.

\section{Reach-only task}

As a control task, the monkeys also learned to perform a reach-only task without grasping the objects. For this motor task, the monkey was required to reach toward the disk and place its hand onto the empty slot on the disk (Figs. $1 \mathrm{~A}$, hand end position; $2 F$ ). The visual stimuli used to cue the monkey (color change of the fixation points) and the timing parameters were exactly the same as during the reach-and-grasp task. After reaching, the monkey was required to leave its open hand on the disk for $530 \mathrm{~ms}$ to receive the juice reward. Optic fibers monitored the position of the hand. This task served as a motor control task to isolate the grasping (and lifting) phase from the reaching phase associated with a reach-andgrasp movement.

\section{Objects}

Since many grasping neurons show specific responses for a particular size and shape of an object (Rizzolatti et al., 1998; Murata et al., 2000; Raos et al., 2006), we used six objects of different sizes or shapes in the motor experiments. These objects included four spheres of different diameters $(16,23,30$, and $40 \mathrm{~mm})$, a cube $12 \mathrm{~mm}$ on a side, and a cylinder of $12 \mathrm{~mm}$ in diameter and $18 \mathrm{~mm}$ in height.

\section{Types of grip used by the macaque monkeys}

Our monkey subjects preferred to grasp the objects with either a "padto-side" grip, pinching the object between the first and second digit (see Fig. 2A,B), a "pad-to-pad"-or precision—grip (see Fig. 2C), or a "thumb-to-second-third" grip (second and third digits in opposition to thumb) (see Fig. 2D) (for an extensive description of the different grips displayed by macaques, see Macfarlane and Graziano, 2009). Even for the largest object used in this study (sphere of $4 \mathrm{~cm}$ diameter), a clear wholehand grasp (also called "hand wrap") was never displayed. This object was mostly grasped from below using the "thumb-to-second-third" grip (see Fig. 2E).

Since monkeys were not trained to perform a whole-hand grasp, and because no detailed kinematics from the hand and fingers were recorded during the actual fMRI sessions, we did not attempt to distinguish among the different types of grasps in the present study.

\section{Scanning}

Functional images were acquired with a 3.0 tesla full-body scanner (TIM Trio; Siemens), using a gradient-echo $\mathrm{T} 2^{\star}$-weighted echo-planar imaging sequence (40 horizontal slices; TR, $2 \mathrm{~s}$; TE, $17 \mathrm{~ms} ; 1.25 \times 1.25 \times 1.25$ $\mathrm{mm}^{3}$ isotropic voxels) with a custom-built eight-channel phased-array receive coil, and a saddle-shaped, radial transmit-only surface coil (Kolster et al., 2009).

Before each monkey scanning session, a contrast agent, monocrystalline iron oxide nanoparticle (MION) (Sinerem; Laboratoire Guerbet), was injected into the femoral/saphenous vein $(6-11 \mathrm{mg} / \mathrm{kg})$. Use of the contrast agent improved the contrast-noise ratio approximately threefold (Vanduffel et al., 2001; Leite et al., 2002) and enhanced spatial selectivity of the MR signal changes (Zhao et al., 2006), compared with blood oxygenation level-dependent (BOLD) measurements. Whereas BOLD measurements depend on cerebral blood volume (CBV), blood flow, and oxygen extraction, MION measurements depend only on blood volume (Mandeville and Marota, 1999). Accordingly, we have inverted the polarity of all signal-change values to account for the difference between MION CBV and BOLD activation maps (increased brain activation produces a decrease in MR signal in MION CBV maps).

Motor tasks were scanned using a block design, with alternating $30 \mathrm{~s}$ blocks (epochs containing 15 brain volumes) of one motor task followed by $30 \mathrm{~s}$ of rest (passive fixation of a centrally positioned red fixation point without moving the hand). During scanning, the same object was presented for a period of $30 \mathrm{~s}$ within the same block or epoch. A typical run consisted of $30 \mathrm{~s}$ each of reach-only task, rest, reach-and-grasp task (with the same object being grasped repeatedly during this block/epoch), rest, reach-only task, rest, reach-and-grasp task (different object than in previous grasping block), and again rest (passive fixation), etc., for a total duration of $480 \mathrm{~s}$ ( 240 volumes). So these runs contained four complete "reach-only-rest-reach-and-grasp-rest" cycles with a total of four different objects (for instance, $16 \mathrm{~mm}$ sphere, $40 \mathrm{~mm}$ sphere, cylinder, cube between glued plastic bars) presented each run. Between runs and sessions, objects on the disk were replaced by other objects. Within a run, monkey subjects performed an equal number of grasping and reach-only movements (M1: mean grasps/run, $34.17 \pm 1.47$; mean reaches/run, $34.5 \pm 1.84 ; \mathrm{M} 2:$ mean grasps/run, $18.17 \pm 1.05$; mean reaches/run, $18.33 \pm 1.26)$.

\section{Volume-based data analysis}

Data were analyzed using statistical parametric mapping (SPM5) and Match software. Only those runs were analyzed in which the monkeys held fixation within the window for $>90 \%$ of the time and which yielded no significant ( $p>0.05, t$ test) between-condition (reach-only, grasping, and rest) differences in number of saccades or SD of the horizontal $(x)$ and vertical $(y)$ components of the eye traces (monkey M1: mean number of saccades $/ \mathrm{min}, 5.50 \pm 1.41, p=0.389 ; \mathrm{SD}(x)=0.54 \pm 0.10$, $p=0.383 ; \operatorname{SD}(y)=0.94 \pm 0.46, p=0.700$; monkey M2: mean number of saccades $/ \mathrm{min}, 9.42 \pm 3.36, p=0.179 ; \mathrm{SD}(x)=1.22 \pm 0.66, p=0.710$ $\mathrm{SD}(y)=2.17 \pm 1.57, p=0.841)$. Realignment parameters were included as covariates of no-interest to remove brain motion artifacts. Brain shifts induced by hand motion were small but evident mostly in the dorsoven$\operatorname{tral}(y)$ translational motion realignment regressor (average shifts in this direction ranged between 0.3 and $0.6 \mathrm{~mm}$ across the whole run). Human fMRI grasping studies using block designs seem to suffer more from artifacts related to hand motion (including both head motion and massinduced magnetic field distortions) and better results have been obtained with event-related designs using widely spaced trials (Culham et al., 2003). The method of head fixation using an MR-compatible head post in our study prevents large shifts in the position of the monkey's head.

Spatial preprocessing consisted of realignment and rigid coregistration with a template anatomy (M12) (Ekstrom et al., 2008). To compensate for echo-planar distortions in the images as well as interindividual anatomical differences, the functional images were warped to the template anatomy using nonrigid matching software, BrainMatch software (Chef d'Hotel et al., 2002). The algorithm computes a dense deformation 
field by the composition of small displacements minimizing a local correlation criterion. Regularization of the deformation field is obtained by low-pass filtering. The functional volumes were then resliced to $1 \mathrm{~mm}^{3}$ isotropic and smoothed with an isotropic Gaussian kernel (full width at half maximum, $1.5 \mathrm{~mm}$ ). Group analyses (fixed effects) were performed with an equal number of volumes per monkey, supplemented with single-subject analysis, and the level of significance was set at $p<0.05$ corrected (familywise error) for multiple comparisons. For display purposes, SPM T-maps were presented on both inflated and flattened representations of M12 anatomical template, using Caret software (version 5.61; http://brainvis.wustl.edu/wiki/index.php/Caret:About).

\section{Region of interest-based analysis}

Statistical significance was tested using MarsBaR (version 0.41.1). The significance threshold for the one-tailed $t$ tests was set at $p<0.05$, Bonferroni corrected for the number of regions of interest (ROIs).

Motor and premotor ROIs. We defined primary motor cortex F1, in addition to several dorsal and ventral premotor sectors, directly onto the template anatomy (M12). These included F2 and F7 in dorsal premotor cortex, F4 and three F5 sectors in ventral premotor cortex, in addition to F3 (supplementary motor area or SMA proper) and F6 (pre-SMA) (Matelli et al., 1985, 1991). The three F5 sectors (Nelissen et al., 2005; Gerbella et al., 2011) correspond to two regions located in the posterior bank of the inferior ramus of the arcuate sulcus (F5p and F5a), and a third region on the convexity posterior to the inferior arcuate ramus (F5c).

Somatosensory ROIs. We defined two ROIs in primary somatosensory cortex $\mathrm{S} 1$, one corresponding to areas $3 \mathrm{a}$ and $3 \mathrm{~b}$, located in the posterior bank of the central sulcus, and a second one corresponding to areas 1 and 2, located on the posterior central gyrus (see Fig. 4). In addition, we defined a ROI termed PEip, corresponding to the portion of area 5 in the medial or upper bank of the rostral intraparietal sulcus (IPS), anterior to area MIP.

In the parietal operculum and upper bank of the lateral sulcus, we delineated a ROI for secondary somatosensory region S2 and for the parietal ventral area (PV) (Krubitzer et al., 1995; Disbrow et al., 2003).

Parietal ROIs. In the intraparietal sulcus, we defined areas AIP and MIP. Delineation of area AIP was based on previous single-cell (Murata et al., 2000) and fMRI studies (Durand et al., 2007). The posterior border of AIP with lateral intraparietal area (LIP) was derived using a saccadic activity task, which yielded saccadic eye movement responses in LIP but not AIP (Durand et al., 2007). Area MIP was delineated based on macaque maps from Lewis and Van Essen (2000). Areas V6A and V6 were identified based on their anatomical locations as proposed by Galletti et al. (1999a,b).

In the inferior parietal lobule (IPL), four regions were delineated, corresponding to PF, PFG, PG, and Opt (Gregoriou et al., 2006; Rozzi et al., 2008; Nelissen et al., 2011). Areas PF and PFG correspond to rostral IPL area $7 \mathrm{~b}$, whereas PG and Opt primarily correspond to caudal area $7 \mathrm{a}$ (Gregoriou et al., 2006).

\section{Time courses}

ROI time courses were made by extracting the raw MR signal from a ROI as a whole (by averaging the signal from all voxels at a certain time point within the ROI) for individual runs, smoothed with a 5 point moving average followed by a linear detrending to remove signal drift across the 8 min runs. Percentage MR signal change was calculated using the rest (fixation-only) epochs as a baseline after removing the first four volumes. Variability between epochs within a run was calculated and represented as dark gray bands flanking the time courses.

In addition, two raw unsmoothed time courses from a single run are also presented.

\section{Results}

The macaque cortical regions responding significantly more strongly (group data, $n=2, p<0.05$, corrected) during the grasping task (Fig. $2 A-E$ ) compared with the reaching control task (Fig. $2 F$ ), are shown in Figure $3 A$ (top view of contralateral
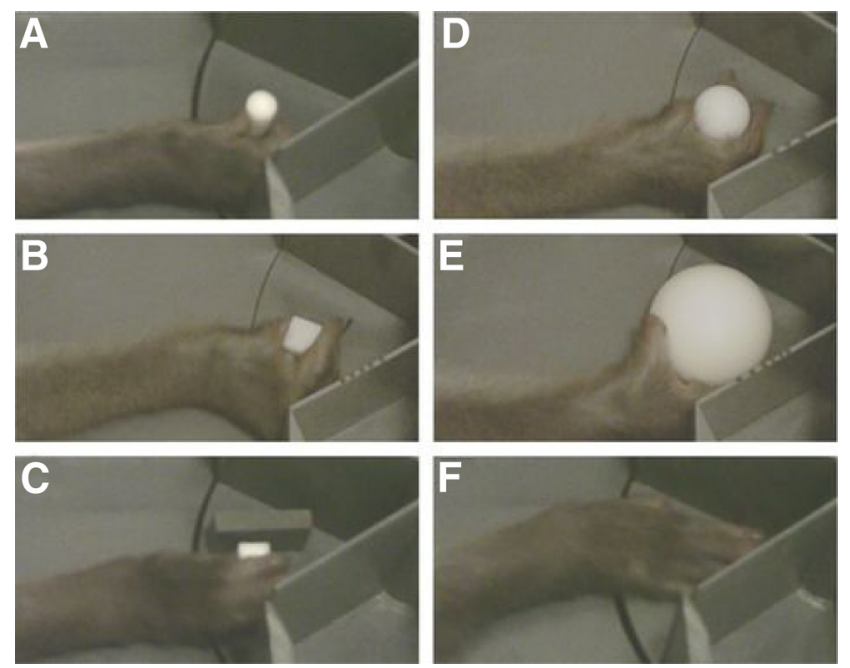

Figure 2. Examples of grasping and reach-only movements. From $\boldsymbol{A}$ to $\boldsymbol{E}$ are shown a monkey grasping a cylinder, a cube, a cube placed between two fixed plastic bars, and two spheres of different sizes (23 and $40 \mathrm{~mm}$ ). F, A monkey reaching and placing its hand on the disk (reach-only task).

and ipsilateral hemisphere) and Figure 3, $B$ and $C$ (semilateral view of contralateral left and ipsilateral right hemisphere).

In the contralateral hemisphere (Figs. $3 A, B$; 4, left panel), grasping versus reaching evoked significant signal changes in portions of premotor F5 (mainly sectors F5p and F5a in the arcuate sulcus) and F4, as well as in a region anterioventral to F5a designated the granular frontal opercular (GrFO) area (Gerbella et al., 2011). In addition, grasping versus reaching revealed larger activity in the hand region of motor area F1, in portions of somatosensory area SI (including area $3 a, 3 b, 1$, and 2), in area 5 (PEip in the upper bank of rostral intraparietal sulcus and PE on the superior parietal lobule convexity) and in area AIP in the rostral lower bank of the IPS and area PFG (part of area 7b) in the rostral portion of the inferior parietal lobule. Finally, several activations were found in the lateral sulcus corresponding to secondary somatosensory cortex SII, parietal ventral area (PV) and additional medial and ventral somatosensory fields, possibly corresponding to ventral somatosensory area (VS) and parietal rostral area (PR) (Krubitzer et al., 1995; Disbrow et al., 2003). Regions activated in the ipsilateral hemisphere (Figs. $3 A, C ; 4$, right panel), included portions of premotor $\mathrm{F} 5$, the hand region of primary motor area F1, area $3 \mathrm{a}$ and $3 \mathrm{~b}$ and portions of IPL areas PFG and PG. In addition, as demonstrated in Figure 4, strong ipsilateral activations were found in secondary somatosensory cortex in the upper bank of the lateral sulcus (and insula), together with additional anterior spots (possibly corresponding to VS and PR). In neither hemisphere were there any early or extrastriate visual areas that were more strongly activated for grasping compared with reaching (Figs. 3, 4). In addition, we performed a ROI analysis for a selection of contralateral cortical regions in both single subjects, thereby plotting the percentage MR signal change for both motor tasks versus the baseline rest (fixation-only) condition (Fig. 5).

In both animals, grasping in the dark (compared with reachonly) yielded significant contralateral activations in motor area F1, premotor regions F4, F5p, and F5a (Fig. 5A,B). In monkey $\mathrm{M} 1$, the F5 sector on the convexity (F5c) and F3 (SMA) also showed a significant grasping-related fMRI response. ROI analysis of several regions within both primary (areas 3a, 3b, 1, and 2) and secondary (SII, PV) somatosensory cortex, in addition to the 
anterior portion of area 5 in the IPS (area PEip), yielded significant responses during grasping compared with reaching in both animals (Fig. 5C,D). Finally, in parietal cortex, the only regions consistently more activated during the grasping versus reaching task (in both animals) included area AIP anteriorly in the IPS and area PFG in the rostral IPL. Areas V6A and MIP (also termed PRR) did not differentiate between the grasping and reaching tasks, showing almost equal increases in MR signal relative to fixation (except for V6A in monkey M1) during execution of either motor task (Fig. $5 E, F$ ), indicating their involvement in the transport phase of the arm during reaching and grasping movements (Nishimura et al., 2007).

In Figure 6, we plot the time courses of activations during typical "reach-onlyrest (fixation-only, no hand/arm movements)-reach-and-grasp-rest" cycles for each of the monkey subjects in several parietal and premotor regions. Figure 6, $A$ and $B$, shows the time courses of key regions involved in grasping control, AIP and the two F5 sectors located in the arcuate sulcus (F5p and F5a). In both subjects, grasping in the dark yielded a significant increase in MR signal compared with reach-only and rest. In Figure 6, $C$ and $D$, we plotted similar time courses for the data from V6A, MIP, and F2. All regions show robust signal increases during the reach-only blocks, as well as during the reach-and-grasp epochs (less clear for V6A of monkey 1). Since it was recently shown (Raos et al., 2004) that the ventrorostral portion of F2 houses a distal motor field for the hand, we also plotted the time course for this subdivision of F2 (termed F2vr). Although the overall fMRI responses from $\mathrm{F} 2 \mathrm{vr}$ are stronger than those of $\mathrm{F} 2$ in both subjects (Fig. 6C,D), ROI analysis of motor responses in F2vr did not yield any significant differences between grasping and reaching in either monkey (M1: $t$ statistic, -0.19 ; $p$ corrected, 0.82 ; M2: $t$ statistic, $0.65 ; p$ corrected, 0.45$)$. Finally, we present the raw (unsmoothed) MR signal for ROIs AIP and F5p from a single run, containing four cycles of "reach-only-rest-reach-and-grasp-rest," for monkey M1 (Fig. 6E), showing clear grasping-related modulations in both areas.

\section{Discussion}

\section{Grasping organization in the brain}

Since the original description of "hand manipulation" neurons in the anterior intraparietal sulcus by Mountcastle et al. (1975), a large body of evidence has accumulated that suggests that parietal area AIP plays a pivotal role in the organization of grasping movements. Single-cell studies (Taira et al., 1990; Sakata et al., 1995; Murata et al., 2000) showed that AIP contains motordominant, visual-dominant, and visuomotor neurons. At the population level, AIP responses code the size, shape, and orientation of an object (also called the "intrinsic" object features) but not the position of the object ("extrinsic" feature). Lesion studies (Gallese et al., 1994) demonstrated that AIP inactivation leads to an inability to preshape the contralateral hand while leaving reaching toward the location of the object intact. In agreement with these observations, AIP displayed a significantly higher grasping-related response compared with reaching in both our subjects. A number of human imaging studies have also described grasping-related responses in the anterior part of the IPS, the possible homolog of macaque AIP (Grafton et al., 1996; Faillenot et al., 1997; Binkofski et al., 1998; Culham et al., 2003; Frey et al., 2005; Begliomini et al., 2007). Furthermore, transcranial magnetic stimulation studies show that a virtual lesion centered over human AIP disrupts hand preshaping (Tunik et al., 2005). Our fMRI data also provide additional evidence for area PFG (within area $7 \mathrm{~b}$ ) playing a role in the execution of grasping movements, as does area AIP. Electrophysiological experiments showed that PFG is a visuomotor, hand-related area (Rozzi et al., 2008), which contains neurons discharging during both the execution and observation of grasping (Fogassi et al., 2005).

Another monkey area involved in organizing grasping is ventral premotor area F5, more specifically the portions of F5 located in the posterior bank of the arcuate sulcus, F5p and F5a (Nelissen et al., 2005; Belmalih et al., 2009; Gerbella et al., 2011). These F5 components have strong connections with area AIP (Luppino et al., 1999; Borra et al., 2008; Gerbella et al., 2011). Whereas F5p contains a hand field and encodes goal-directed hand actions such as grasping or manipulating objects (Raos et al., 2006; Umiltà et al., 2007), F5c, located on the convexity, is a hand and mouth field containing motor as well as visuomotor neurons. It has been suggested that $\mathrm{F} 5 \mathrm{c}$ plays a role in several cognitive motor functions, such as interpreting the goal of observed motor acts or evaluating sensory information for perceptual decisions (Gallese 


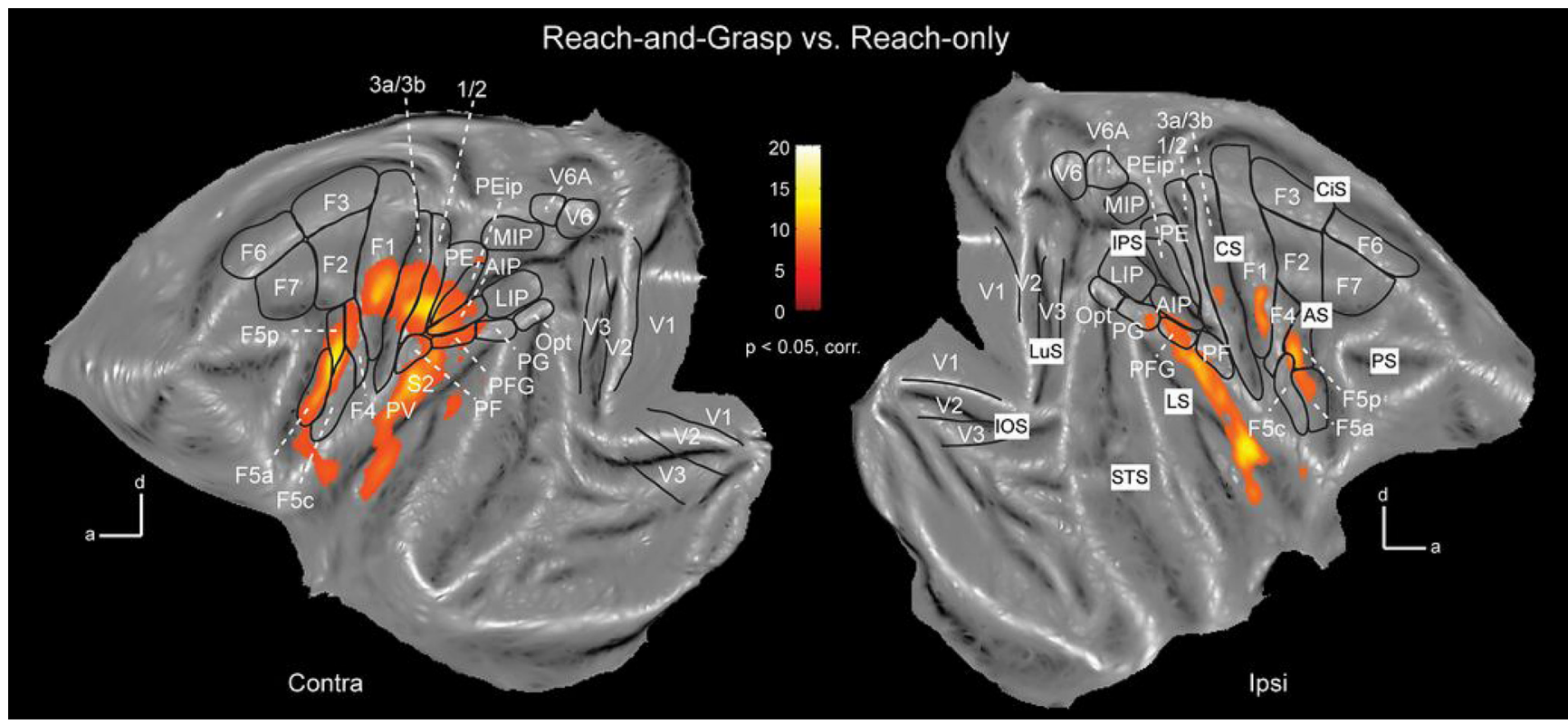

Figure 4. Flat maps showing grasping-related fMRI activations. Flat maps showing grasping-related fMRI responses ( $p<0.05$, corrected) in contralateral (left) and ipsilateral (right) hemispheres. The black outlines indicate regions of interest. Shown are group data $(n=2)$. CiS, Cingulate sulcus. Other conventions are as in Figure 3.
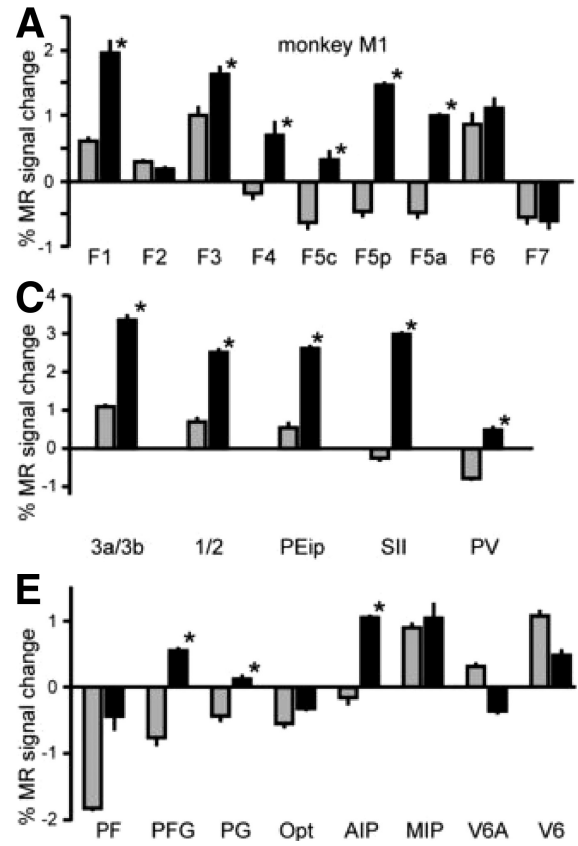
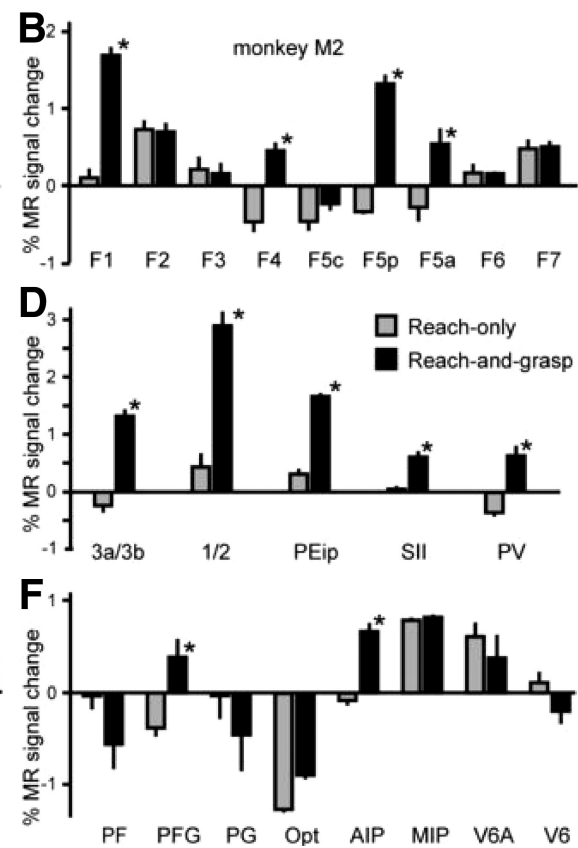

Figure 5. ROl analysis. $\boldsymbol{A}-\boldsymbol{F}$, Plots showing percentage MR signal change for both monkey subjects (M1:A, $C, E ; M 2: B, D, F)$ for reach-only (gray) and grasping (black) compared with rest (fixation-only) for several ROls in contralateral motor and premotor cortex $(\boldsymbol{A}, \boldsymbol{B})$, primary and secondary somatosensory cortex $(\boldsymbol{C}, \boldsymbol{D})$, and parietal cortex $(\boldsymbol{E}, \boldsymbol{F})$. The asterisks indicate significantly $(p<0.05$, corrected) stronger responses for grasping compared with reaching. Errors on the histogram plots indicate variability across runs.

et al., 1996; Rizzolatti and Craighero, 2004; Romo et al., 2004; Wardak et al., 2010). Inactivation experiments show clear impairment of hand shaping after muscimol injections into F5 components located in the caudal bank of the arcuate sulcus, with the effect of small injections being confined to the contralesional hand but with larger inactivations also affecting the ipsilateral hand (Fogassi et al., 2001). In agreement with the abovementioned single-cell and lesion data, we observed significant grasping-related responses in contralateral and ipsilateral F5.
These premotor fMRI responses also fit with human imaging studies showing grasping-related responses in ventral premotor cortex during either haptic object manipulation (Binkofski et al., 1999), grasping in the dark (Ehrsson et al., 2000), or visually guided grasping (Toni et al., 2001; Cavina-Pratesi et al., 2010).

As expected, grasping yielded significant fMRI signal increases in the hand sector of F1 (Grafton et al., 1996; Faillenot et al., 1997; Culham et al., 2003; Begliomini et al., 2007; Nishimura et al., 2007) because of the individual finger movement control associated with the grasping task, which are not present in the reach-only task.

Grasping in the dark also activated several regions in primary (areas $3 a, 3 b, 1,2$ ) and secondary (SII) somatosensory cortex, in addition to portions of area 5 (especially PEip in the medial bank of rostral IPS). This activity is probably linked to tactile feedback necessary for grasping and the control of finger grip force, which might be especially enhanced when vision is not available to guide the hand (Gregoriou et al., 2005). Because of the design of our motor tasks and since detailed hand kinematics were not recorded, it is difficult to attribute the differential fMRI responses (grasping vs reaching) in somatosensory cortex to either preshaping of the hand or to the actual contact of the fingers with the object. Previous single-cell data indicate a role for areas 5 and $7 \mathrm{~b}$ in grasping (Gardner et al., 2007), with SI (mainly area 2) being the possible source of somatosensory feedback to area 5, given the strong connections between the two areas (Pearson and Powell, 1985). Furthermore, macaque studies showed that, whereas SI neurons dis- 
A
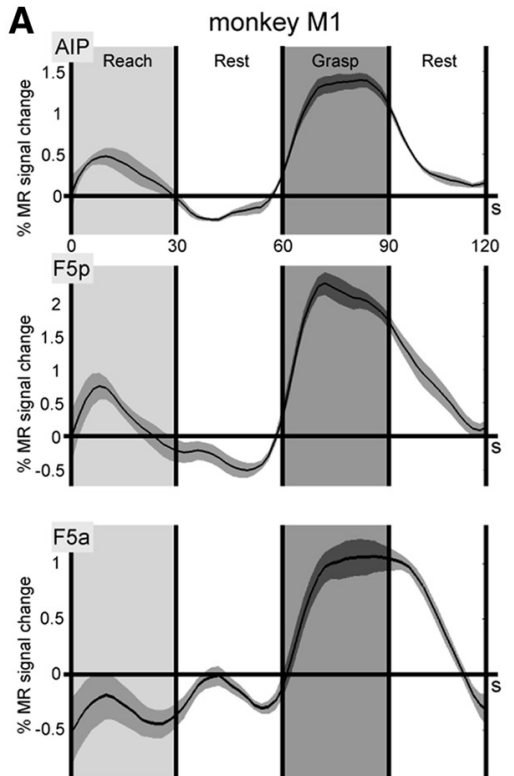

C
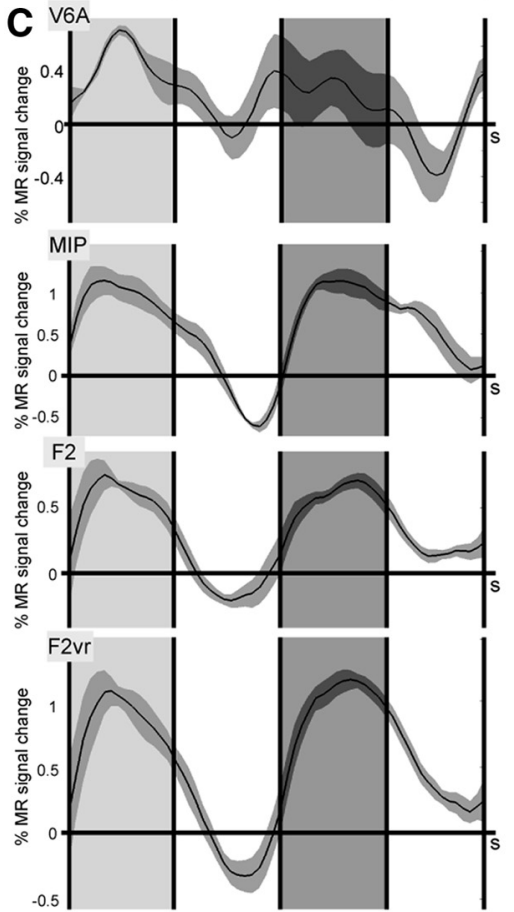

E

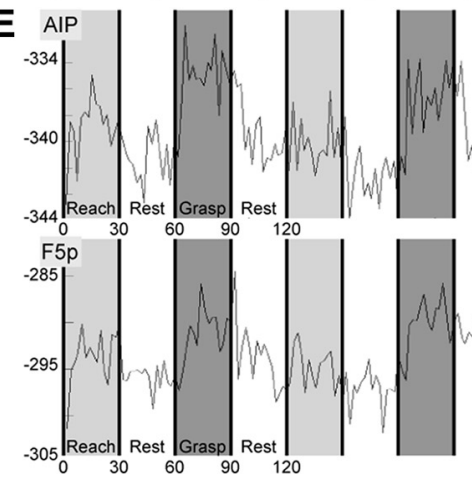

B
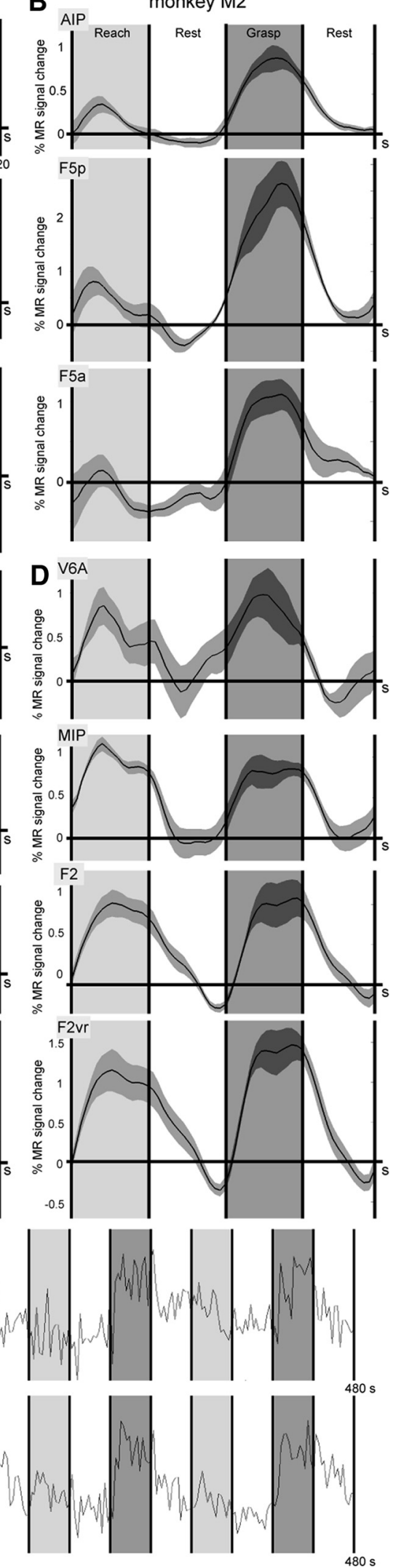

Figure 6. Time courses of MR signal in grasping and reaching circuit. $\boldsymbol{A}, \boldsymbol{B}$, Time courses plotting percentage MR signal change for both subjects (M1, $\boldsymbol{A} ; \mathrm{M} 2, \boldsymbol{B})$ in lateral parietofrontal grasping circuit (areas AIP and F5p/F5a) over a 2 min period consisting of 30 s each of reach-only (gray), rest, grasping (black), and again rest. The horizontal axis represents time in seconds. The gray bands flanking the time courses indicate variability between epochs. C, D, Time courses plotting percentage MR signal change for both subjects (M1, C; M2, D) in the medial parietofrontal reaching circuit (areas V6A, MIP, and F2) as well as in F2vr (distal field of F2) charged most strongly during hand contact, lifting, and holding, neurons in areas 5 and 7 responded most strongly during the hand preshaping phase before object contact (Debowy et al., 2001).

Somatosensory information is sent from SI to areas 5 and 7, but also ventrally to SII. SII has a complex organization, with partitioning schemes varying from two up to seven different regions (Krubitzer et al., 1995; Disbrow et al., 2003; Fitzgerald et al., 2004; Eickhoff et al., 2006). The observation that SII and PV respond bilaterally, unlike SI (Disbrow et al., 2000), fits with our monkey fMRI data, in which grasping in the dark yielded strong bilateral activations in the upper bank of the lateral sulcus and insula (Disbrow et al., 2003; Hinkley et al., 2007). SII neurons become active during object manipulation (Krubitzer et al., 1995; Fitzgerald et al., 2004) and are involved in tactile object recognition (Reed et al., 2004). The existence of strong connections between SII and AIP (Borra et al., 2008) and between SII and F5a (Gerbella et al., 2011) suggest that SII is a possible candidate for the source of tactile feedback to both AIP and F5, for the selection of the appropriate grasping scheme or for the control of finger grip force.

\section{Separate circuits for grasping and reaching?}

Although previous neurophysiological and lesion data (Gallese et al., 1994; Binkofski et al., 1998; Fogassi et al., 2001; Culham et al., 2003; Rice et al., 2006) in both humans and monkeys suggested that reaching and grasping are mediated by two separate anatomical pathways (Jeannerod, 1981; Jeannerod et al., 1995), a strict dichotomy between a lateral grasping versus a medial reaching circuit is probably too simplistic. Recent evidence shows that the medial parietofrontal reaching circuit is also involved in wrist orientation and grip formation (Raos et al., 2004; Fattori et al., 2009, 2010), in addition to playing an important role in organizing reaching toward the location of an object (Colby and Duhamel, 1991;

over a 2 min period consisting of 30 s each of reach-only (gray), rest, grasping (black), and rest. Other conventions are the same as in $\boldsymbol{A}$ and $\boldsymbol{B}$. $\boldsymbol{E}$, Raw time course of a single run for AIP and $F 5 p$ of $M 1$, showing four cycles of reach-only-rest-reachand-grasp-rest. During rest, the monkey kept its gaze on the central fixation point, while leaving its hand in the start position without moving. The numbers on the horizontal axis indicate time in seconds; the numbers on the $y$-axis indicate arbitrary scanner units. 
Snyder et al., 1997; Fattori et al., 2001; Galletti et al., 2003; Bosco et al., 2010). Furthermore, Raos et al. (2003) showed that F2 contains both a proximal and distal forelimb field, the latter sector (F2vr) coding particularly wrist movements, which suggests a role for F2vr in grasping (Raos et al., 2004). In addition, Battaglini et al. (2002) showed that lesions of V6A have an effect on both reaching and grasping (abnormal wrist orientation and flexion) in monkeys.

Why, then, did we fail to see clear grasping-related activity in V6A and $\mathrm{F} 2$, in contrast to robust $\mathrm{PMRI}$ grasping-related responses in AIP and F5? One explanation might be that the motor tasks in our study were performed in complete darkness. The objects were never illuminated and monkeys executed their grasps based on both haptic information and memory. Typically, human fMRI studies that find grasping-related responses in dorsal premotor cortex have compared grasping with reaching under visual guidance (Grol et al., 2007). This fits with the monkey electrophysiology study by Raos et al. (2004), showing grasping-related responses in F2, especially under visual guidance. Second, our grasping task required very little change in wrist orientation to grasp and lift the objects. As previous studies have shown, both V6A and F2 grasping neurons play important roles in wrist orientation during grasping (Raos et al., 2004; Fattori et al., 2010). Note that human fMRI studies comparing grasping versus reaching rarely find grasping-related activation in the possible human homolog of V6A, the superior parieto-occipital cortex (SPOC) (Cavina-Pratesi et al., 2010), except when the grasping task requires changes in wrist orientation (Culham et al., 2003). Third, our block design paradigm required repetitive reaching and grasping movements. Several studies suggest that the dorsomedial reaching circuit can contribute to grasping depending on the degree of on-line control required by the type of prehension movement (Gréa et al., 2002; Grol et al., 2007; Verhagen et al., 2008). Future fMRI grasping studies in the monkey comparing grasping executed in light and in darkness, as well as using motor tasks with less stereotyped grasping movements requiring more pronounced wrist orientations, might shed additional light on the role of the dorsomedial reaching circuit in wrist control and grasping.

Although the monkey model has tremendously aided our understanding of the organization of grasping, many aspects of this complex motor behavior still need to be investigated. For instance, it is not yet known in detail at what level the ventral stream (coding the meaning of objects) and the parietal and frontal cortex (where decision-making takes place) interact with the grasping network (Rizzolatti and Luppino, 2001). Furthermore, the exact role of the somatosensory cortex in providing haptic or memory-related feedback and its interaction with the basal ganglia for finger grip force control is still far from clear. Whole-brain imaging techniques like monkey fMRI might prove useful for investigating these issues since they allow investigators to visualize motor task-related activations throughout the brain and to compare these with both anatomical (diffusion tensor imaging) and effective (electrical stimulation) connectivity maps (Ekstrom et al., 2008) from the same animal and suggest guidelines for possible future electrophysiological recordings performed simultaneously in multiple grasping-activated areas.

\section{Notes}

Supplemental material for this article is available at http://neuroserv.med. kuleuven.be/downloadarea/stimuli.php. Two supplemental movies show examples of the reach-and-grasp and reach-only task: Nelissen_grasping task.mp4 and Nelissen_reaching_task.mp4. This material has not been peer reviewed.

\section{References}

Battaglini PP, Muzur A, Galletti C, Skrap M, Brovelli A, Fattori P (2002) Effects of lesions to area V6A in monkeys. Exp Brain Res 144:419-422.

Begliomini C, Wall MB, Smith AT, Castiello U (2007) Differential cortical activity for precision and whole-hand visually guided grasping in humans. Eur J Neurosci 25:1245-1252.

Belmalih A, Borra E, Contini M, Gerbella M, Rozzi S, Luppino G (2009) Multimodal architectonic subdivision of the rostral part (area F5) of the macaque ventral premotor cortex. J Comp Neurol 512:183-217.

Binkofski F, Dohle C, Posse S, Stephan KM, Hefter H, Seitz RJ, Freund HJ (1998) Human anterior intraparietal area subserves prehension: a combined lesion and functional MRI activation study. Neurology 50:1253-1259.

Binkofski F, Buccino G, Stephan KM, Rizzolatti G, Seitz RJ, Freund HJ (1999) A parieto-premotor network for object manipulation: evidence from neuroimaging. Exp Brain Res 128:210-213.

Borra E, Belmalih A, Calzavara R, Gerbella M, Murata A, Rozzi S, Luppino G (2008) Cortical connections of the macaque anterior intraparietal (AIP) area. Cereb Cortex 18:1094-1111.

Bosco A, Breveglieri R, Chinellato E, Galletti C, Fattori P (2010) Reaching activity in the medial posterior parietal cortex of monkeys is modulated by visual feedback. J Neurosci 30:14773-14785.

Brochier T, Umiltà MA (2007) Cortical control of grasp in non-human primates. Curr Opin Neurobiol 17:637-643.

Buneo CA, Jarvis MR, Batista AP, Andersen RA (2002) Direct visuomotor transformations for reaching. Nature 416:632-636.

Castiello U, Begliomini C (2008) The cortical control of visually guided grasping. Neuroscientist 14:157-170.

Cavina-Pratesi C, Monaco S, Fattori P, Galletti C, McAdam TD, Quinlan DJ, Goodale MA, Culham JC (2010) Functional magnetic resonance imaging reveals the neural substrates of arm transport and grip formation in reach-to-grasp actions in humans. J Neurosci 30:10306-10323.

Chef d'Hotel C, Hermosillo G, Faugeras O (2002) Flows of diffeomorphisms for multimodal image registration. Proc IEEE Int $S$ Bio Im 7-8:753.

Colby CL, Duhamel JR (1991) Heterogeneity of extrastriate visual areas and multiple parietal areas in the macaque monkey. Neuropsychologia 29:517-537.

Connolly JD, Andersen RA, Goodale MA (2003) FMRI evidence for a "parietal reach region" in the human brain. Exp Brain Res 153:140-145.

Culham JC, Valyear KF (2006) Human parietal cortex in action. Curr Opin Neurobiol 16:205-212.

Culham JC, Danckert SL, DeSouza JF, Gati JS, Menon RS, Goodale MA (2003) Visually guided grasping produces fMRI activation in dorsal but not ventral stream brain areas. Exp Brain Res 153:180-189.

Debowy DJ, Ghosh S, Ro JY, Gardner EP (2001) Comparison of neuronal firing rates in somatosensory and posterior parietal cortex during prehension. Exp Brain Res 137:269-291.

Disbrow E, Roberts T, Krubitzer L (2000) Somatotopic organization of cortical fields in the lateral sulcus of Homo sapiens: evidence for SII and PV. J Comp Neurol 418:1-21.

Disbrow E, Litinas E, Recanzone GH, Padberg J, Krubitzer L (2003) Cortical connections of the second somatosensory area and the parietal ventral area in macaque monkeys. J Comp Neurol 462:382-399.

Durand JB, Nelissen K, Joly O, Wardak C, Todd JT, Norman JF, Janssen P, Vanduffel W, Orban GA (2007) Anterior regions of monkey parietal cortex process visual 3D shape. Neuron 55:493-505.

Ehrsson HH, Fagergren A, Jonsson T, Westling G, Johansson RS, Forssberg H (2000) Cortical activity in precision- versus power-grip tasks: an fMRI study. J Neurophysiol 83:528-536.

Eickhoff SB, Schleicher A, Zilles K, Amunts K (2006) The human parietal operculum. I. Cytoarchitectonic mapping of subdivisions. Cereb Cortex 16:254-267.

Ekstrom LB, Roelfsema PR, Arsenault JT, Bonmassar G, Vanduffel W (2008) Bottom-up dependent gating of frontal signals in early visual cortex. Science 321:414-417.

Faillenot I, Toni I, Decety J, Grégoire MC, Jeannerod M (1997) Visual pathways for object-oriented action and object recognition: functional anatomy with PET. Cereb Cortex 7:77-85. 
Fattori P, Gamberini M, Kutz DF, Galletti C (2001) “Arm-reaching” neurons in the parietal area V6A of the macaque monkey. Eur J Neurosci 13:2309-2313.

Fattori P, Breveglieri R, Marzocchi N, Filippini D, Bosco A, Galletti C (2009) Hand orientation during reach-to-grasp movements modulates neuronal activity in the medial posterior parietal area V6A. J Neurosci 29:1928-1936.

Fattori P, Raos V, Breveglieri R, Bosco A, Marzocchi N, Galletti C (2010) The dorsomedial pathway is not just for reaching: grasping neurons in the medial parieto-occipital cortex of the macaque monkey. J Neurosci 30:342-349.

Filimon F (2010) Human cortical control of hand movements: parietofrontal networks for reaching, grasping, and pointing. Neuroscientist 16:388-407.

Fitzgerald PJ, Lane JW, Thakur PH, Hsiao SS (2004) Receptive field properties of the macaque second somatosensory cortex: evidence for multiple functional representations. J Neurosci 24:11193-11204.

Fogassi L, Gallese V, Buccino G, Craighero L, Fadiga L, Rizzolatti G (2001) Cortical mechanism for the visual guidance of hand grasping movements in the monkey: a reversible inactivation study. Brain 124:571-586.

Fogassi L, Ferrari PF, Gesierich B, Rozzi S, Chersi F, Rizzolatti G (2005) Parietal lobe: from action organization to intention understanding. Science 308:662-667.

Frey SH, Vinton D, Norlund R, Grafton ST (2005) Cortical topography of human anterior intraparietal cortex active during visually guided grasping. Brain Res Cogn Brain Res 23:397-405.

Gallese V, Murata A, Kaseda M, Niki N, Sakata H (1994) Deficit of hand preshaping after muscimol injection in monkey parietal cortex. Neuroreport 5:1525-1529.

Gallese V, Fadiga L, Fogassi L, Rizzolatti G (1996) Action recognition in the premotor cortex. Brain 119:593-609.

Galletti C, Fattori P, Kutz DF, Gamberini M (1999a) Brain location and visual topography of cortical area V6A in the macaque monkey. Eur J Neurosci 11:575-582.

Galletti C, Fattori P, Gamberini M, Kutz DF (1999b) The cortical visual area V6: brain location and visual topography. Eur J Neurosci 11:3922-3936.

Galletti C, Kutz DF, Gamberini M, Breveglieri R, Fattori P (2003) Role of the medial parieto-occipital cortex in the control of reaching and grasping movements. Exp Brain Res 153:158-170.

Gardner EP, Babu KS, Reitzen SD, Ghosh S, Brown AS, Chen J, Hall AL, Herzlinger MD, Kohlenstein JB, Ro JY (2007) Neurophysiology of prehension. I. Posterior parietal cortex and object-oriented hand behaviors. J Neurophysiol 97:387-406.

Gerbella M, Belmalih A, Borra E, Rozzi S, Luppino G (2011) Cortical connections of the anterior (F5a) subdivision of the macaque ventral premotor area F5. Brain Struct Funct 216:43-65.

Grafton ST (2010) The cognitive neuroscience of prehension: recent developments. Exp Brain Res 204:475-491.

Grafton ST, Fagg AH, Woods RP, Arbib MA (1996) Functional anatomy of pointing and grasping in humans. Cereb Cortex 6:226-237.

Gréa H, Pisella L, Rossetti Y, Desmurget M, Tilikete C, Grafton S, Prablanc C, Vighetto A (2002) A lesion of the posterior parietal cortex disrupts on-line adjustments during aiming movements. Neuropsychologia 40:2471-2480.

Gregoriou GG, Savaki HE (2003) When vision guides movement: a functional imaging study of the monkey brain. Neuroimage 19:959-967.

Gregoriou GG, Luppino G, Matelli M, Savaki HE (2005) Frontal cortical areas of the monkey brain engaged in reaching behavior: a ${ }^{14} \mathrm{C}-$ deoxyglucose imaging study. Neuroimage 27:442-464.

Gregoriou GG, Borra E, Matelli M, Luppino G (2006) Architectonic organization of the inferior parietal convexity of the macaque monkey. J Comp Neurol 496:422-451.

Grol MJ, Majdandzić J, Stephan KE, Verhagen L, Dijkerman HC, Bekkering H, Verstraten FA, Toni I (2007) Parieto-frontal connectivity during visually guided grasping. J Neurosci 27:11877-11887.

Hinkley LB, Krubitzer LA, Nagarajan SS, Disbrow EA (2007) Sensorimotor integration in S2, PV, and parietal rostroventral areas of the human sylvian fissure. J Neurophysiol 97:1288-1297.

Jeannerod M (1981) Intersegmental coordination during reaching at natu- ral visual objects. In: Attention and performance IX (Long J, Baddeley A, eds), pp 153-168. Hillsdale, NJ: Erlbaum.

Jeannerod M, Decety J, Michel F (1994) Impairment of grasping movements following a bilateral posterior parietal lesion. Neuropsychologia 32:369-380.

Jeannerod M, Arbib MA, Rizzolatti G, Sakata H (1995) Grasping objects: the cortical mechanisms of visuomotor transformation. Trends Neurosci 18:314-320.

Kalaska JF, Scott SH, Cisek P, Sergio LE (1997) Cortical control of reaching movements. Curr Opin Neurobiol 7:849-859.

Kolster H, Mandeville JB, Arsenault JT, Ekstrom LB, Wald LL, Vanduffel W (2009) Visual field map clusters in macaque extrastriate visual cortex. J Neurosci 29:7031-7039.

Krubitzer L, Clarey J, Tweedale R, Elston G, Calford M (1995) A redefinition of somatosensory areas in the lateral sulcus of macaque monkeys. J Neurosci 15:3821-3839.

Leite FP, Tsao D, Vanduffel W, Fize D, Sasaki Y, Wald LL, Dale AM, Kwong KK, Orban GA, Rosen BR, Tootell RB, Mandeville JB (2002) Repeated fMRI using iron oxide contrast agent in awake, behaving macaques at 3 tesla. Neuroimage 16:283-294.

Lewis JW, Van Essen DC (2000) Mapping the architectonic subdivisions in the macaque monkey, with emphasis on parieto-occipital cortex. J Comp Neurol 428:79-111.

Luppino G, Murata A, Govoni P, Matelli M (1999) Largely segregated parietofrontal connections linking rostral intraparietal cortex (areas AIP and VIP) and the ventral premotor cortex (areas F5 and F4). Exp Brain Res 128:181-187.

Macfarlane NB, Graziano MS (2009) Diversity of grip in Macaca mulatta. Exp Brain Res 197:255-268.

Mandeville JB, Marota JJ (1999) Vascular filters of functional MRI: spatial localization using BOLD and CBV contrast. Magn Reson Med 42:591-598.

Matelli M, Luppino G, Rizzolatti G (1985) Patterns of cytochrome oxidase activity in the frontal agranular cortex of the macaque monkey. Behav Brain Res 18:125-136.

Matelli M, Luppino G, Rizzolatti G (1991) Architecture of superior and mesial area 6 and the adjacent cingulate cortex in the macaque monkey. J Comp Neurol 311:445-462.

Mountcastle VB, Lynch JC, Georgopoulos A, Sakata H, Acuna C (1975) Posterior parietal association cortex of the monkey: command functions for operations within extrapersonal space. J Neurophysiol 38:871-908.

Murata A, Gallese V, Luppino G, Kaseda M, Sakata H (2000) Selectivity for the shape, size, and orientation of objects for grasping in neurons of monkey parietal area AIP. J Neurophysiol 83:2580-2601.

Nelissen K, Luppino G, Vanduffel W, Rizzolatti G, Orban GA (2005) Observing others: multiple action representation in the frontal lobe. Science 310:332-336.

Nelissen K, Vanduffel W, Orban GA (2006) Charting the lower superior temporal (LST) region, a new motion sensitive region in monkey STS. J Neurosci 26:5929-5947.

Nelissen K, Borra E, Gerbella M, Rozzi S, Luppino G, Vanduffel W, Rizzolatti G, Orban GA (2011) Action observation circuits in the macaque monkey cortex. J Neurosci 31:3743-3756.

Nishimura Y, Onoe H, Morichika Y, Tsukada H, Isa T (2007) Activation of parieto-frontal stream during reaching and grasping studied by positron emission tomography in monkeys. Neurosci Res 59:243-250.

Pearson RC, Powell TP (1985) The projection of the primary somatic sensory cortex on area 5 in the monkey. Brain Res 356:89-107.

Prado J, Clavagnier S, Otzenberger H, Scheiber C, Kennedy H, Perenin MT (2005) Two cortical systems for reaching in central and peripheral vision. Neuron 48:849-858.

Raos V, Franchi G, Gallese V, Fogassi L (2003) Somatotopic organization of the lateral part of area F2 (dorsal premotor cortex) of the macaque monkey. J Neurophysiol 89:1503-1518.

Raos V, Umiltá MA, Gallese V, Fogassi L (2004) Functional properties of grasping-related neurons in the dorsal premotor area F2 of the macaque monkey. J Neurophysiol 92:1990-2002.

Raos V, Umiltá MA, Murata A, Fogassi L, Gallese V (2006) Functional properties of grasping-related neurons in the ventral premotor area F5 of the macaque monkey. J Neurophysiol 95:709-729. 
Reed CL, Shoham S, Halgren E (2004) Neural substrates of tactile object recognition: an fMRI study. Hum Brain Mapp 21:236-246.

Rice NJ, Tunik E, Grafton ST (2006) The anterior intraparietal sulcus mediates grasp execution, independent of requirement to update: new insights from transcranial magnetic stimulation. J Neurosci $26: 8176-8182$.

Rizzolatti G, Craighero L (2004) The mirror neuron system. Annu Rev Neurosci 27:169-192.

Rizzolatti G, Luppino G (2001) The cortical motor system. Neuron 31:889-901.

Rizzolatti G, Camarda R, Fogassi L, Gentilucci M, Luppino G, Matelli M (1988) Functional organizationof inferior area 6 in the macaque monkey. II. Area F5 and the control of distal movements. Exp Brain Res 71:491-507.

Rizzolatti G, Luppino G, Matelli M (1998) The organization of the cortical motor system: new concepts. Electroencephalogr Clin Neurophysiol 106:283-296.

Romo R, Hernández A, Zainos A (2004) Neuronal correlates of a perceptual decision in ventral premotor cortex. Neuron 41:165-173.

Rozzi S, Ferrari PF, Bonini L, Rizzolatti G, Fogassi L (2008) Functional organization of inferior parietal lobule convexity in the macaque monkey: electrophysiological characterization of motor, sensory and mirror responses and their correlation with cytoarchitectonic areas. Eur J Neurosci 28:1569-1588.

Sakata H, Taira M, Murata A, Mine S (1995) Neural mechanisms of visual guidance of hand action in the parietal cortex of the monkey. Cereb Cortex 5:429-438.

Snyder LH, Batista AP, Andersen RA (1997) Coding of intention in the posterior parietal cortex. Nature 386:167-170.
Stoianovici D, Patriciu A, Petrisor D, Mazilu D, Kavoussi L (2007) A new type of motor: pneumatic step motor. IEEE ASME Trans Mechatron 12:98-106.

Taira M, Mine S, Georgopoulos AP, Murata A, Sakata H (1990) Parietal cortex neurons of the monkey related to the visual guidance of hand movement. Exp Brain Res 83:29-36.

Toni I, Rushworth MF, Passingham RE (2001) Neural correlates of visuomotor associations. Spatial rules compared with arbitrary rules. Exp Brain Res 141:359-369.

Tunik E, Frey SH, Grafton ST (2005) Virtual lesions of the anterior intraparietal area disrupt goal-dependent on-line adjustments of grasp. Nat Neurosci 8:505-511.

Umiltà MA, Brochier T, Spinks RL, Lemon RN (2007) Simultaneous recording of macaque premotor and primary motor cortex neuronal populations reveals different functional contributions to visuomotor grasp. J Neurophysiol 98:488-501.

Vanduffel W, Fize D, Mandeville JB, Nelissen K, Van Hecke P, Rosen BR, Tootell RB, Orban GA (2001) Visual motion processing investigated using contrast agent-enhanced fMRI in awake behaving monkeys. Neuron 32:565-577.

Verhagen L, Dijkerman HC, Grol MJ, Toni I (2008) Perceptuo-motor interactions during prehension movements. J Neurosci 28:4726-4735.

Wardak C, Vanduffel W, Orban GA (2010) Searching for a salient target involves frontal regions. Cereb Cortex 20:2464-2477.

Zhao F, Wang P, Hendrich K, Ugurbil K, Kim SG (2006) Cortical layerdependent BOLD and CBV responses measured by spin-echo and gradient-echo fMRI: insights into hemodynamic regulation. Neuroimage 30:1149-1160. 